\title{
Forced downregulation of RACK1 inhibits glioma development by suppressing Src/Akt signaling activity
}

\author{
RENJUN PENG, BING JIANG, JIANRONG MA, ZHIMING MA, XIN WAN, \\ HONGWEI LIU, ZIGUI CHEN, QUAN CHENG and RUI CHEN
}

\author{
Department of Neurosurgery, Xiangya Hospital of Central South University, Changsha, Hunan 410078, P.R. China
}

Received June 12, 2013; Accepted August 14, 2013

DOI: $10.3892 /$ or.2013.2723

\begin{abstract}
Glioma is the most common primary brain malignant tumor. Receptor for activated C-kinase 1 (RACK1) is widely expressed in the central nervous system, and regulates multiple cellular processes including cell survival, proliferation, migration and metastasis. However, the role of RACK1 in glioma has never been revealed. The present study, for the first time, showed that RACK1 expression was significantly higher in glioma tissues and cell lines when compared with that in normal brain tissues, and was positively associated with the malignancy of glioma. siRNA-induced RACK1 downregulation significantly suppressed the proliferation and invasion of human glioma U87 and CHG-5 cells, while it promoted their apoptosis by upregulating Bax expression and reducing $\mathrm{Bcl}-2$ expression. Furthermore, forced downregulation of RACK1 notably inhibited tumor xenograft growth in nude mice. These findings suggest that RACK1 plays a critical role in the development and progression of glioma in vitro and in vivo. Moreover, siRNA-induced RACK1 downregulation markedly reduced the activity of Src/Akt signaling pathway, which plays an important role in the growth and behavior of human malignancies, indicating that siRNA-mediated RACK1 downregulation inhibited glioma probably via suppressing Src/Akt signaling activity. The present study highlighted the role of RACK1 in glioma, and demonstrated that RACK1 is a novel promising therapeutic target for glioma treatment.
\end{abstract}

\section{Introduction}

Malignant glioma is the most common primary brain tumor, accounting for approximately $80 \%$ of malignant tumors in the central nervous system. It has a very poor prognosis, mainly due to its resistance to radiotherapy, chemotherapy

Correspondence to: Professor Bing Jiang, Department of Neurosurgery, Xiangya Hospital of Central South University, 87 Xiangya Road, Changsha, Hunan 410078, P.R. China

E-mail: csujiangbing@163.com

Key words: glioma, receptor for activated C-kinase 1, proliferation, apoptosis, invasion and adjuvant therapies (1-3). Generally, patients diagnosed with glioblastoma, the most malignant form of glioma, live approximately only 1 year after diagnosis (4). Despite the marked developments in the therapy of other types of cancer, the median survival rate of malignant glioma has not improved in the past years (5). Therefore, a more effective therapeutic strategy is urgently required.

Receptor for activated C-kinase 1 (RACK1) is a member of the intracellular receptors for activated protein kinase $\mathrm{C}$ (PKC) (6). RACK1 is widely expressed in the central nervous system, and is involved in multiple crucial cellular process including cell growth, proliferation, apoptosis and migration (7-9). In recent years, accumulating evidence has shown that RACK1 plays an important role in various types of cancer (10-12). Therefore, we hypothesized that RACK1 may act as an important regulator in the development and progression of malignant glioma.

In this study, we first determined the mRNA and protein expression levels of RACK1 in glioma tissues of different grades as well as in normal brain tissues. Then, the mRNA and protein expression of RACK1 was determined in two types of human glioma cell lines U87 and CHG-5. Using RACK1-specific siRNA, the effects as well as the molecular mechanism of RACK1 downregulation involved in proliferation, apoptosis and invasion of U87 and CHG-5 cells were further investigated in vitro and in vivo.

\section{Materials and methods}

Materials and reagents. The protocol used in the present study was approved by the Ethics Committee of Central South University. Written informed consent was obtained from each participant. Forty-five glioma tissues (10 cases of WHO I, 13 cases of WHO II, 12 cases of WHO III, and 10 cases of WHO IV) were obtained from patients who underwent surgery from October 2011 to October 2012 at the Department of Neurosurgery, Xiangya Hospital of Central South University (Hunan, China), and 10 normal brain tissues from patients with cerebral trauma were used as controls. The human glioma U87 (WHO IV) and CHG-5 (WHO II) cell lines were obtained from the Cell Bank of Central South University. Dulbecco's modified Eagle's medium (DMEM), opti-MEM, fetal bovine serum (FBS) and Lipofectamine 2000 transfection agent were purchased from Invitrogen Life Technologies (USA). MTT was 
obtained from Sigma (USA). The bicinchoninic acid (BCA) protein assay kit was purchased from Pierce Biotechnology, Inc. (USA). PVDF membrane was obtained from Pall (USA). All siRNAs and antibodies were obtained from Santa Cruz Biotechnology, Inc. (USA). The Annexin V-FITC Apoptosis Detection kit was purchased from Biovision (USA). Matrigel was purchased form BD Biosciences (USA).

Cell culture. U87 and CHG-5 cell lines were cultured in DMEM containing $10 \% \mathrm{FBS}, 100 \mathrm{U} / \mathrm{ml}$ penicillin and $100 \mathrm{U} / \mathrm{ml}$ streptomycin. Cells were incubated at $37^{\circ} \mathrm{C}$ in a humidified incubator of $95 \%$ air and $5 \% \mathrm{CO}_{2}$.

Real-time RT-PCR. The RACK1 mRNA expression in tissues or cell lines was detected by real-time RT-PCR (ABI 7500). Specific primers for different genes in this study were synthesized by BGI Company (Guangzhou, China). Specific primers for RACK1 were: sense, 5'-GAGCAAATGACCCTTCGT-3' and antisense, 5'-GTAGTGCCCGTTGTGAGA-3'. Specific primers for Bax were: sense, 5'-CCCGAGAGGTCTTTTTCC GAG-3' and antisense, 5'-CCAGCCCATGATGGTTCTGAT-3'. Specific primers for Bcl-2 were: sense, 5'-GGTGGGGTCAT GTGTGTGG-3' and antisense, 5'-CGGTTCAGGTACTCAG TCATCC-3'. Human glyceraldehyde-3-phosphate dehydrogenase (GAPDH) primers were used as a control (sense, 5'-GGCA GCCCAGAACATCATCC-3' and antisense, 5'-GCCAGCCC AAGCATCAAAG-3'). All amplifications were performed in 3 parallel samples.

Western blotting. Cells were lysed by cold RIPA lysis, and the protein concentrations were determined using BCA protein assay kit. Then, proteins of $20 \mu \mathrm{g} /$ lane were loaded on $12 \%$ SDS-PAGE to separate, and then electrophoretically transferred to PVDF membranes. Proteins on the membranes were then probed using primary antibodies according to the supplier's protocol. Following incubation with secondary antibodies, results were visualized with peroxidase and an enhanced chemiluminescence system (Pierce Biotechnology, Inc.) and quantified by Quantity One software (Bio-Rad, USA).

siRNA interference. Human glioma U87 and CHG-5 cells were seeded at a density of $10^{5}$ cells/well in 6-well plates and cultured in DMEM containing 10\% FBS. After incubating at $37^{\circ} \mathrm{C}, 5 \% \mathrm{CO}_{2}$ for $24 \mathrm{~h}, \mathrm{U} 87$ and CHG-5 cells were transfected with siRNA and Lipofectamine 2000 according to the supplier's instruction. Briefly, $100 \mathrm{nmol}$ siRNA and $5 \mu \mathrm{l}$ Lipofectamine 2000 were diluted in opti-MEM to a final volume of $800 \mu \mathrm{l}$. After mixing for $20 \mathrm{~min}$ at room temperature, the siRNA/Lipofectamine 2000 mixture was added. Cells were incubated at $37^{\circ} \mathrm{C} 5 \% \mathrm{CO}_{2}$ for $6 \mathrm{~h}$. Following incubation, the mixture was replaced with DMEM containing $10 \%$ FBS for $24 \mathrm{~h}$.

Proliferation assay. Cell proliferation was determined by MTT assay. After $24 \mathrm{~h}$ post-transfection, the transfection medium in each well was replaced by DMEM medium containing $10 \%$ FBS used before, and was cultured for 12, 24, 36, 48 and $60 \mathrm{~h}$. Then, the medium was replaced by $100 \mu 1$ of fresh serum-free medium and cultured with $0.5 \mathrm{~g} / 1$ MTT. Following incubation at $37^{\circ} \mathrm{C}$ for $4 \mathrm{~h}$, the MTT medium was removed by aspiration and $50 \mu 1$ of DMSO was added to each well. Following incubation at $37^{\circ} \mathrm{C}$ for a further $10 \mathrm{~min}$, the A540 of each sample was measured using a plate reader.

Apoptosis analysis. Flow cytometry was used to determine the cell apoptosis with the Annexin V-FITC Apoptosis Detection kit. After $24 \mathrm{~h}$ post-transfection, cells were harvested and washed with cold PBS twice. Subsequently, $10^{6}$ cells were resuspended in $200 \mu \mathrm{l}$ binding buffer supplemented with $10 \mu \mathrm{l}$ Annexin V-FITC and $5 \mu 1 \mathrm{PI}-\mathrm{PE}$, and incubated in the dark for $30 \mathrm{~min}$. Then, $300 \mu \mathrm{l}$ binding buffer was added followed by flow cytometry assay.

Transwell matrix penetration assay. Cells $\left(10^{5}\right)$ of different groups in $200 \mu \mathrm{l}$ serum-free DMEM were plated on the upper chamber plated on the top side of polycarbonate Transwell filter coated with Matrigel and incubated at $37^{\circ} \mathrm{C}$ for $24 \mathrm{~h}$. Subsequently, cells inside the upper chamber were removed. Cells on the lower membrane surface were fixed in $1 \%$ paraformaldehyde, stained with $0.1 \%$ crystal violet and counted.

Tumorigenesis in nude mice. All animal experiments were approved by the Animal Care and Use Committee of Central South University. RACK1-specific shRNA lentivirus, or non-specific shRNA lentivirus, were purchased from Santa Cruz Biotechnology, Inc. Ten million U87 cells, which were without any treatment or infected with RACK1-specific shRNA lentivirus, or non-specific shRNA lentivirus, were injected subcutaneously into 8 -week old nude mice $(n=5)$, which were maintained under pathogen-limited conditions. The xenograft tumors were measured at 10, 15, 20, 25 and 30 days. We calculated the tumor volume according to $\mathrm{V}(\mathrm{mm})=\mathrm{D} 2\left(\mathrm{~mm}^{2}\right) \mathrm{X} \mathrm{L}(\mathrm{mm}) / 2$, where $\mathrm{D}$ is the smallest perpendicular tumor diameters while $\mathrm{L}$ is the largest perpendicular tumor diameters. The animals were sacrificed at 30 days after implantation. All tumors were photographed and weighed.

Statistical analysis. Data are presented as means \pm standard deviation (SD). SPSS 17.0 software was used to perform statistical analyses using a two-tailed Student's t-test or one-way ANOVA. $P<0.05$ was considered to indicate statistically significant differences. All experiments were repeated at least 3 times.

\section{Results}

Upregulation of RACK1 in human glioma tissues and glioma cell lines. To preliminarily investigate the relationship between RACK1 expression and glioma, we first examined the mRNA and protein expression levels of RACK1 in normal brain tissues, gliomas of different grades and 2 glioma cell lines. As shown in Fig. 1A and B, real-time PCR and western blotting results showed that the expression of RACK1 showed an increasing tendency with the malignancy of glioma. We further determined the expression of RACK1 in 2 human glioma cell lines, U87 (grade IV) and CHG-5 (grade II). As shown in Fig. 1C and D, real-time PCR and western blotting results demonstrated that RACK1 expression was also upregulated in U87 and CHG-5 cells compared to normal brain tissues $(\mathrm{P}<0.01)$. However, there was no difference of RACK1 expres- 


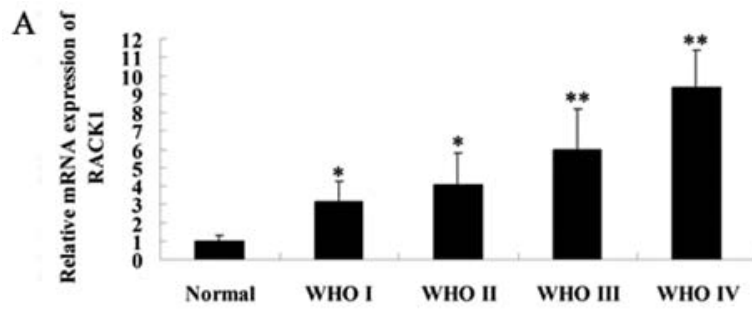

B
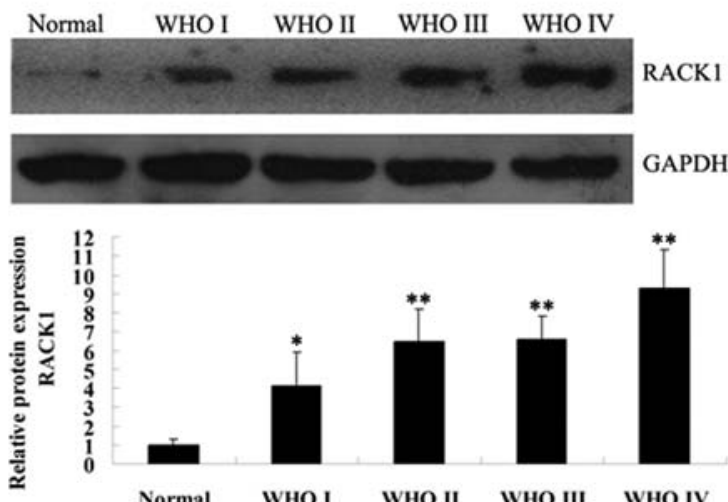

$\mathrm{C}$

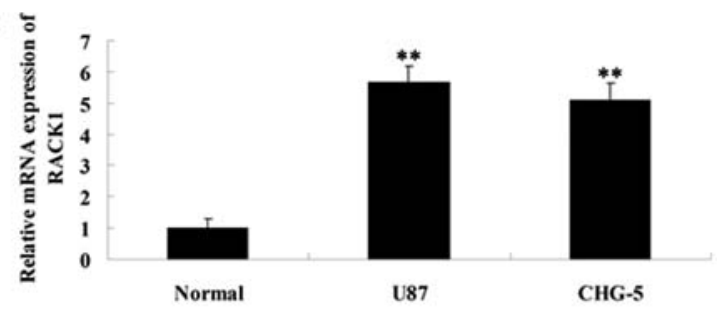

$\mathrm{D}$
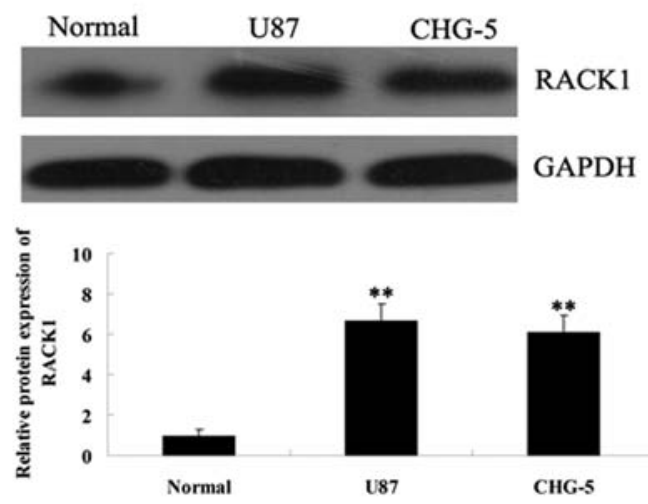

Figure 1. The mRNA and protein expression of RACK1 in glioma tissues and cells. (A) Real-time RT-PCR was performed to determine the relative mRNA expression of RACK1 in gliomas of different grades. (B) Western blotting was applied to determine the protein expression of RACK1 in gliomas of different grades. (C) Real-time RT-PCR was performed to determine the relative mRNA expression of RACK1 in 2 glioma cell lines, U87 and CHG-5. (D) Western blotting was applied to determine the protein expression of RACK1 in 2 glioma cell lines, U87 and CHG-5. All data in the experiment groups were compared with the normal group. ${ }^{*} \mathrm{P}<0.05 ;{ }^{* *} \mathrm{P}<0.01$. RACK1, receptor for activated $\mathrm{C}$-kinase 1 .

sion between these 2 glioma cell lines $(\mathrm{P}>0.05)$. Accordingly, these data above indicate that the increased expression of RACK1 may be associated with the malignancy of glioma.

siRNA-induced RACK1 downregulation in U87 and CHG-5 cells. Due to the increased expression of RACK1 in glioma tissues and cell lines, we used RACK1-specific siRNA to downregulate the expression of RACK1 in U87 and CHG-5 cells to further investigate the role of RACK1 in glioma. After transfection, we first determined the mRNA and protein expression of RACK1 in U87 and CHG-5 cells using real-time RT-PCR and western blotting, respectively. As shown in Fig. 2, after transfection with RACK1-specific siRNA, the mRNA and protein levels of RACK1 in U87 and CHG-5 cells were effectively downregulated $(\mathrm{P}<0.01)$, when compared with those in the untreated (control) and the non-specific siRNA group (NC).

siRNA-induced RACK1 downregulation suppresses proliferation of glioma U87 and CHG-5 cells. To further investigate the role of RACK1 in glioma cells in vitro, MTT assay was performed to determine the effect of siRNA-induced RACK1 downregulation on proliferation of U87 and CHG-5 cells. As shown in Fig. 3, MTT assay demonstrated that in RACK1downregulated U87 and CHG-5 cells, the cell proliferation rate was lower when compared with controls $(\mathrm{P}<0.01)$. These results suggest that RACK1 promotes proliferation of human glioma cells.

siRNA-induced RACK1 downregulation enhances apoptosis of glioma U87 and CHG-5 cells. We further investigated the effect of RACK1 downregulation on apoptosis of glioma cells. As shown in Fig. 4, in RACK1-downregulated U87 and CHG-5
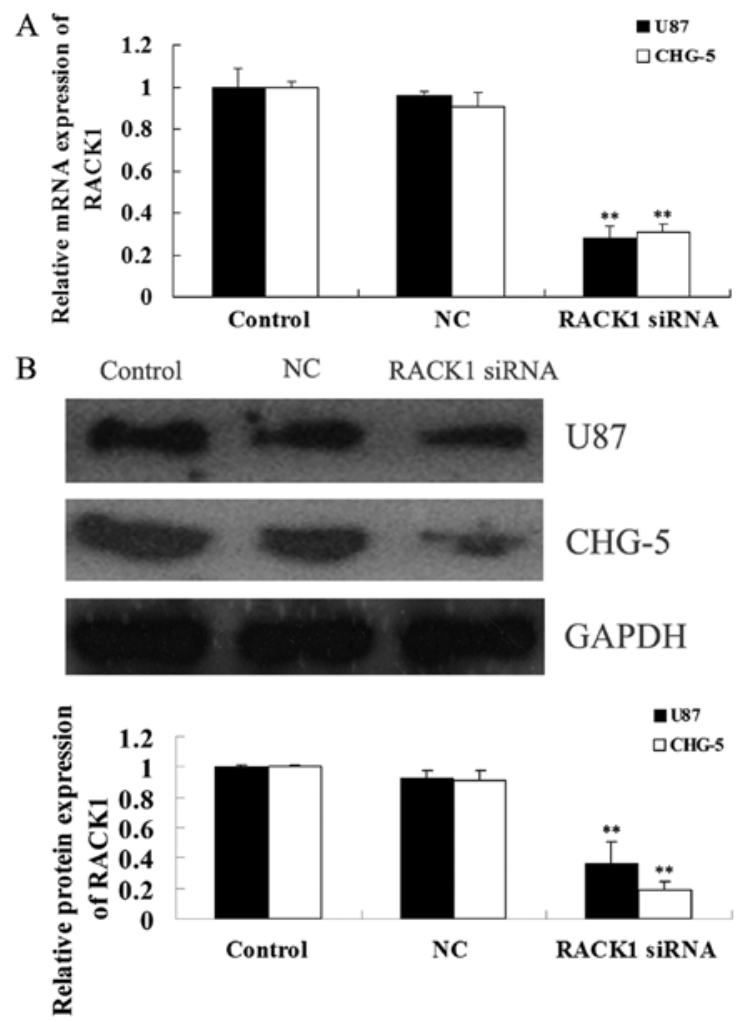

Figure 2. The mRNA and protein expression of RACK1 in U87 and CHG-5 cells after siRNA-mediated interference. Control, cells without any treatment. NC, cells transfected with non-specific siRNA. RACK1 siRNA, cells transfected with RACK1-specific siRNA. (A) Real-time RT-PCR was performed to determine the relative mRNA expression of RACK1 in U87 and CHG-5 cells after siRNA-mediated interference. (B) Western blotting was applied to determine the protein expression of RACK1 in U87 and CHG-5 cells after siRNA-mediated interference. All data in the experiment groups were compared with the control group. ${ }^{* *} \mathrm{P}<0.01$. RACK1, receptor for activated $\mathrm{C}$-kinase 1 . 

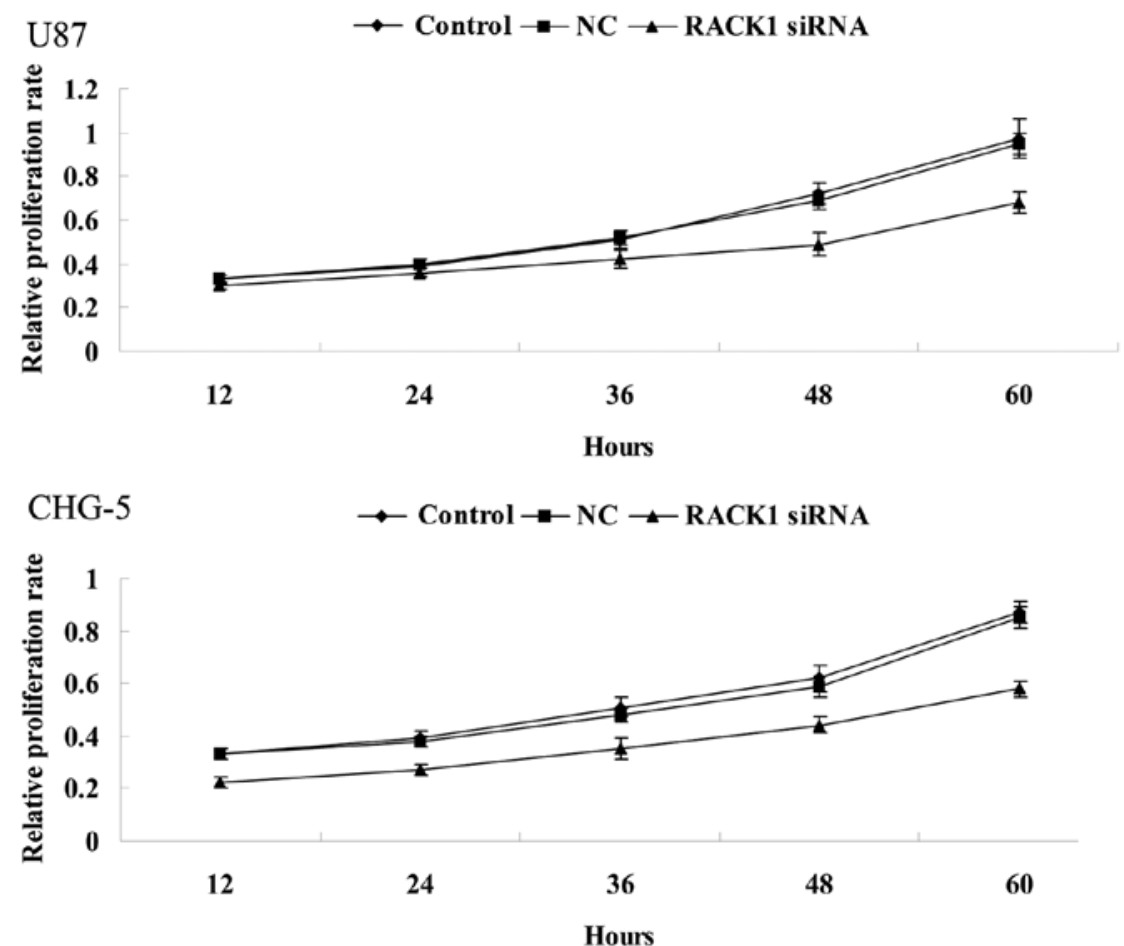

Figure 3. The relative proliferation rate of U87 and CHG-5 cells after siRNA-mediated RACK1 downregulation. After transfection with RACK1-specific siRNA or non-specific siRNA in U87 and CHG-5 cells, which were then cultured for 12, 24, 36, 48 and $60 \mathrm{~h}$, MTT was performed to determine the effect of RACK1 downregulation on cell proliferation. Control, cells without any treatment. NC, cells transfected with non-specific siRNA. RACK1 siRNA, cells transfected with RACK1-specific siRNA. RACK1, receptor for activated C-kinase 1.

A
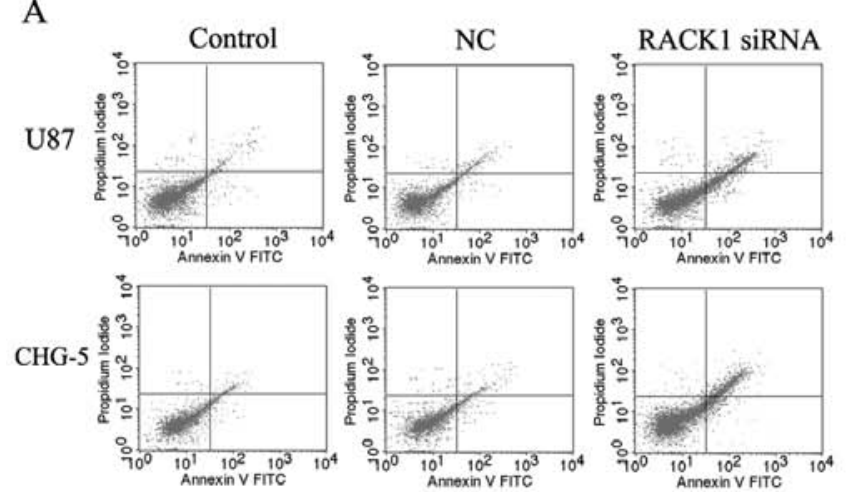

B
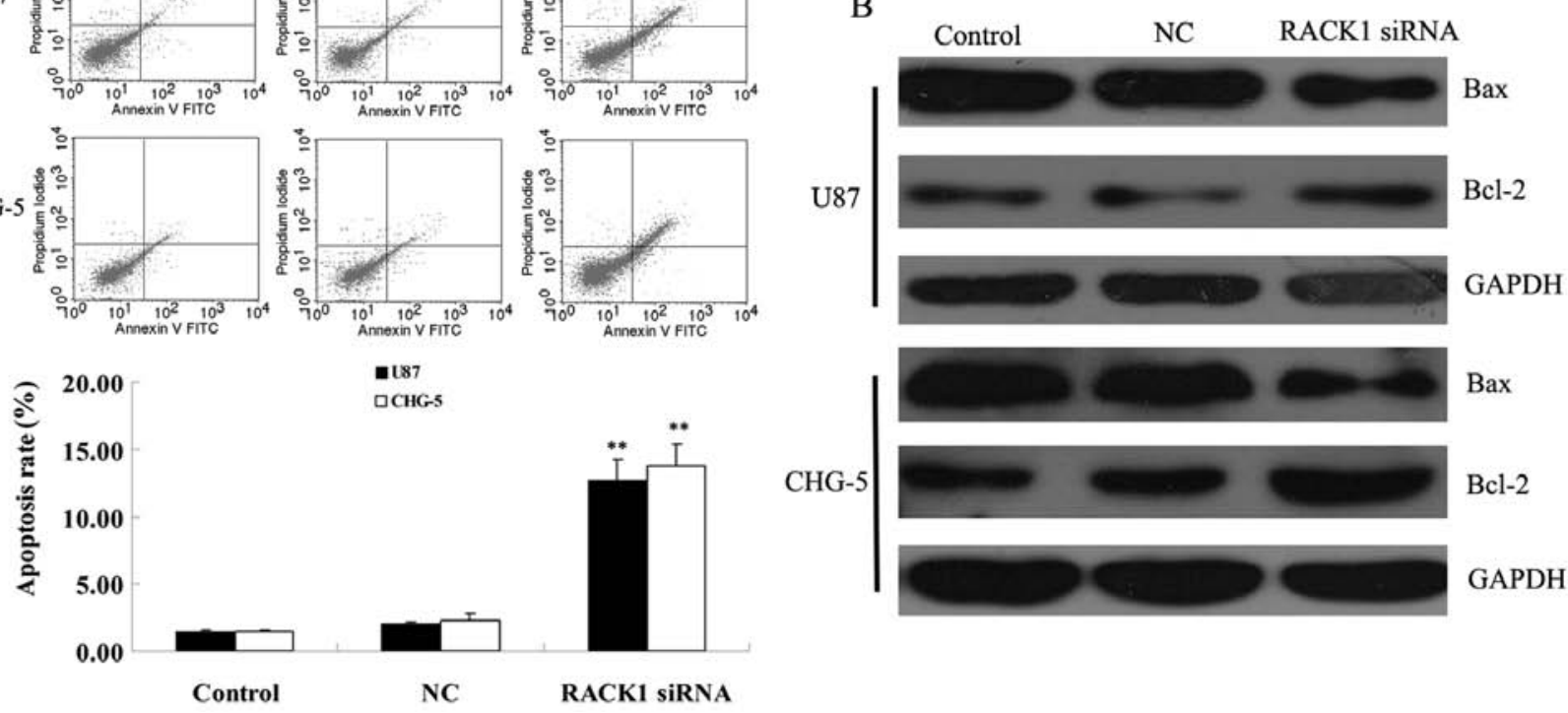

Figure 4. Apoptosis of U87 and CHG-5 cells after siRNA-mediated RACK1 downregulation. Control, cells without any treatment. NC, cells transfected with non-specific siRNA. RACK1 siRNA, cells transfected with RACK1-specific siRNA. (A) Flow cytometry was used to determine the apoptotic rate of U87 and CHG-5 cells after forced downregulation of RACK1. (B) Western blotting was applied to determine the protein expression of Bax and Bcl-2 in U87 and CHG-5 cells after siRNA-mediated RACK1 downregulation. All data in the experiment groups were compared with the control group. ${ }^{* *} \mathrm{P}<0.01$. RACK1, receptor for activated C-kinase 1.

cells, the cell apoptosis rate was much higher when compared with controls $(\mathrm{P}<0.01)$, suggesting that RACK1 may play an inhibitory role in the regulation of apoptosis of human glioma cells. We then examined the expression of apoptotic-related genes in each group. Real-time PCR assay showed that the mRNA expression of pro-apoptotic gene Bax was upregulated, 


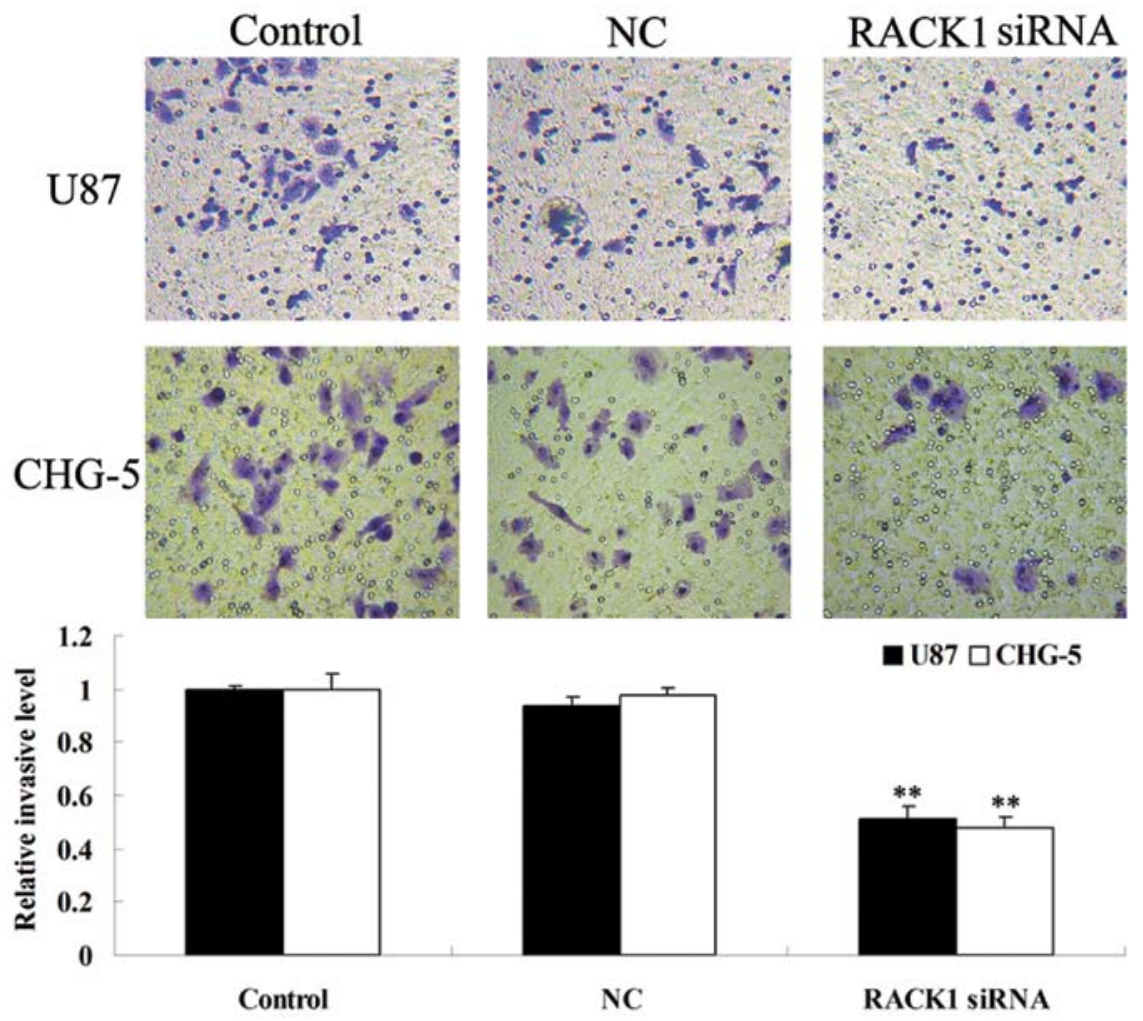

Figure 5. The invasion of U87 and CHG-5 cells after siRNA-mediated RACK1 downregulation. Control, cells without any treatment. NC, cells transfected with non-specific siRNA. RACK1 siRNA, cells transfected with RACK1-specific siRNA. Transwell assay was performed to examine the invasive capacity of U87 and CHG-5 cells after siRNA-mediated RACK1 downregulation. All data in the experiment groups were compared with the control group. ${ }^{* *} \mathrm{P}<0.01$. RACK1, receptor for activated C-kinase 1.

while the mRNA expression of anti-apoptotic gene Bcl-2 was reduced, in RACK1-downregulated glioma U87 and CHG-5 cells, when compared with those in controls. These results indicated that RACK1 may suppress apoptosis of glioma cells in vitro through directly or indirectly regulating the expression of apoptotis-related genes, Bax and Bcl-2.

siRNA-induced RACK1 downregulation inhibits invasion of glioma U87 and CHG-5 cells. The alternations of cell invasion ability of human glioma U87 and CHG-5 cells were examined after transfection with RACK1-specific siRNA. As shown in Fig. 5, RACK1-downregulated cells showed decreased invasion ability when compared with controls $(\mathrm{P}<0.05)$. These data indicate that RACK1 promotes invasion ability of human glioma cells in vitro.

siRNA-induced RACK1 downregulation suppresses survival, apoptosis, migration, and proliferation relative signaling pathways in U87 and CHG-5 cells. Bax is a pro-apoptotic gene, while Bcl-2 has an anti-apoptotic function. It has been well established that the ratio of $\mathrm{Bax} / \mathrm{Bcl}-2$ protein plays a crucial role in regulating cell apoptosis. Hence, we applied real-time RT-PCR to determine the mRNA expression of Bax and Bcl-2 in U87 and CHG-5 cells of each group. As shown in Fig. 6A, the relative mRNA level of Bax was significantly upregulated in U87 and CHG-5 cells after transfection with RACK1-specific siRNA, when compared with that in the control group $(\mathrm{P}<0.01)$. However, the relative mRNA level of Bcl-2 was decreased in U87 and CHG-5 cells after transfec- tion with RACK1-specific siRNA, compared with that in the control group $(\mathrm{P}<0.01)$. These data partly explain the above findings that forced downregulation of RACK1 promotes the apoptosis of U87 and CHG-5 cells.

Src/Akt signaling pathway plays a crucial role in the regulation of survival, proliferation and migration of multiple types of cells. It has been reported that RACK1 induces colon cell apoptosis, partly by suppressing Src activity in Akt pathway. However, whether a similar molecular mechanism exists in glioma cells remains unknown. Thus, we determined the activity of Src/Akt signaling pathway in RACK1-downregulated U87 and $\mathrm{CHG}-5$ cells. As shown in Fig. 6B, the phosphorylation levels of Src and Akt were much lower in U87 and CHG-5 cells after transfection with RACK1-specific siRNA, when compared with those in controls $(\mathrm{P}<0.05)$. These results indicate that the effects of RACK1 on the survival, proliferation, invasion of glioma cells may be involved in the regulation of the Src/Akt signal pathway.

Forced downregulation of RACK1 suppresses tumor xenograft growth in nude mice. To further investigate the role of RACK1 in vivo, a tumor xenograft animal model was conducted using U87 cells, in which RACK1 was successfully knocked down by lentivirus infection. After all animals were subcutaneously implanted with the infected tumor cells, the size of tumors in the RACK1-specific shRNA group was significantly smaller than that in the control and NC group (Fig. 7A). As shown in Fig. 7B, the average tumor weight in the RACK1-specific shRNA group was markedly lower than that in the control and 


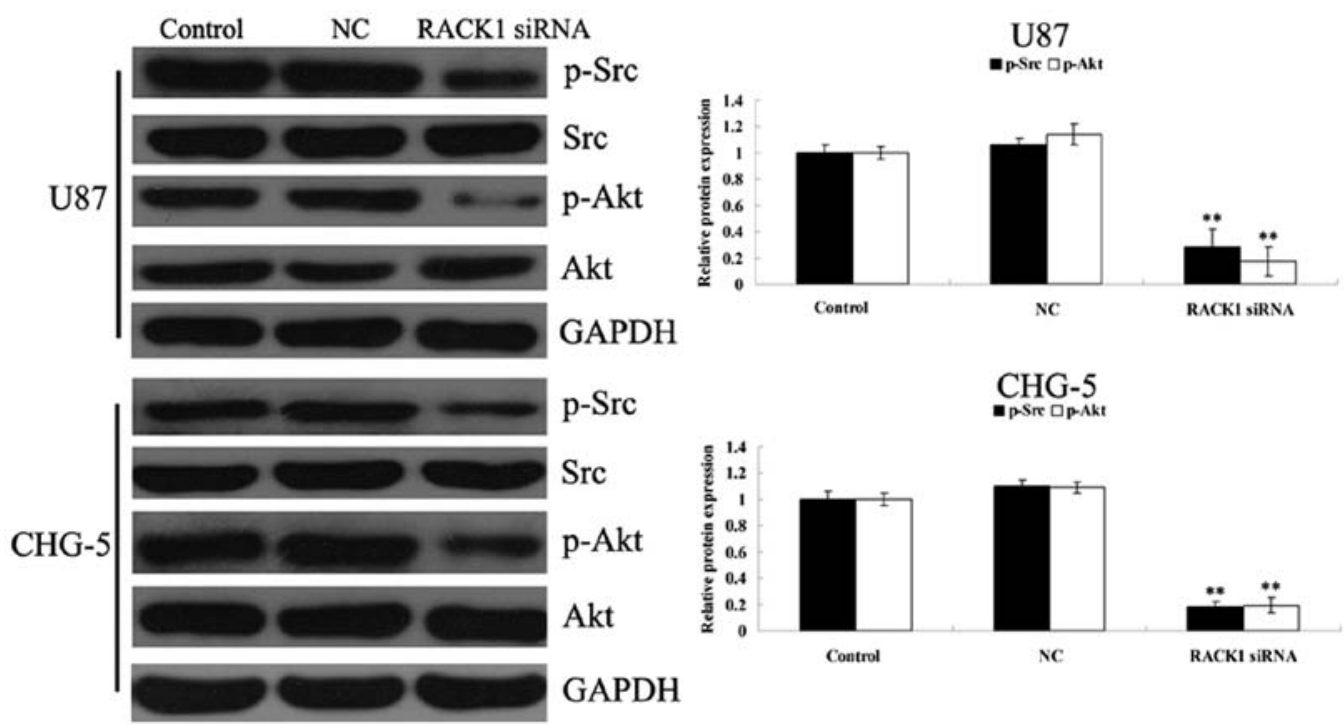

Figure 6. The activity of Src/Akt signaling pathway in U87 and CHG-5 cells after siRNA-mediated RACK1 downregulation. Western blotting was applied to determine the phosphorylation levels of Bax and Bcl-2 proteins in U87 and CHG-5 cells after siRNA-mediated RACK1 downregulation. Control, cells without any treatment. NC, cells transfected with non-specific siRNA. RACK1 siRNA, cells transfected with RACK1-specific siRNA. All data in the experiment groups were compared with the control group. ${ }^{* *} \mathrm{P}<0.01$. RACK1, receptor for activated C-kinase 1.

A $\rightarrow-$ Control -- NC-LV $\rightarrow$ RACK1 shRNA-LV

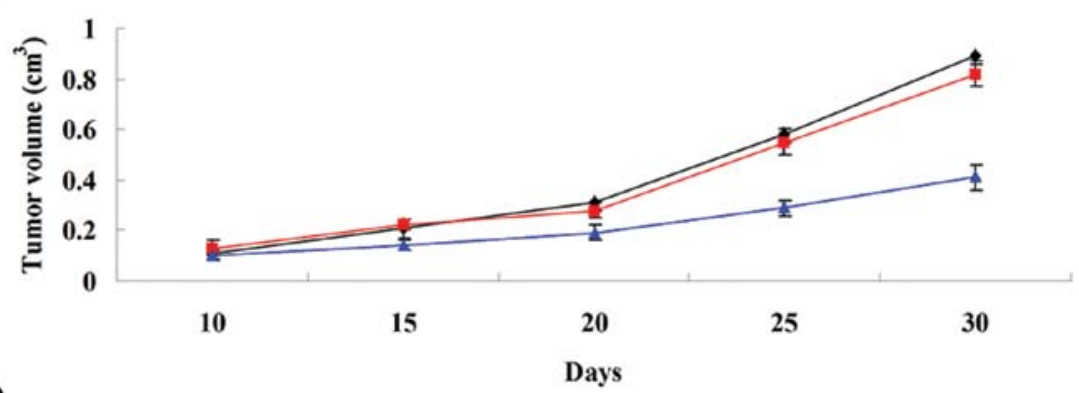

B
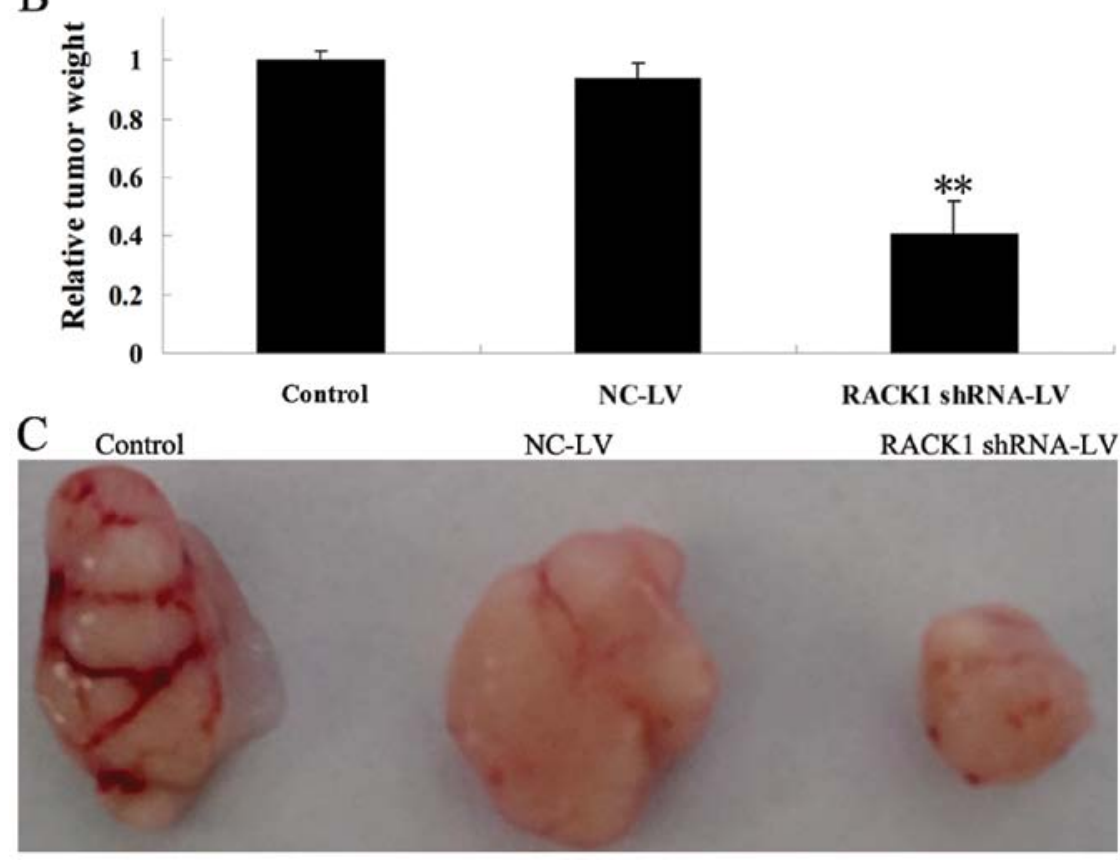

Figure 7. Forced downregulation of RACK1 suppresses tumor xenograft growth in nude mice. The tumor xenograft animal model was conducted using U87 cells, in which RACK1 was successfully knocked down by lentivirus infection. Control, U87 cells without any treatment. NC-LV, U87 cells infected with non-specific shRNA lentivirus. RACK1 shRNA-LV, U87 cells infected with RACK1-specific shRNA lentivirus. (A) The average tumor volume in each group was determined at day 10,15, 20,25 and 30 after implantation. (B) The relative tumor weight in each group after excision. (C) The image of typical tumor in each group after excision. All data in the experiment groups were compared with the control group. ${ }^{* *} \mathrm{P}<0.01$. 
NC group. Moreover, the tumor size in the RACK1-specific shRNA group was much smaller than that in the control and NC group (Fig. 7C). These results are consistent with those of the cell proliferation assay, indicating that forced downregulation of RACK1 significantly inhibits tumor growth, and that RACK1 plays a positive regulatory role in glioma growth in vivo.

\section{Discussion}

As a receptor for activated $\mathrm{PKC}, \mathrm{RACK} 1$ has effects on multiple cellular processes, such as cell proliferation, apoptosis and migration (7-9). As a result, its dysregulation may contribute to tumorigenesis. In fact, RACK1 has been demonstrated to be involved in various types of malignant tumors including hepatocellular carcinoma, lung cancer, colon cancer, prostate cancer, cervical carcinoma, oral squamous cell carcinoma and neuroblastoma (13-19). However, whether RACK1 plays a role in malignant glioma as well as the mechanism involved remains largely unknown.

In the present study, we first examined the expression of RACK1 in glioma tissues of different grades as well as 2 glioma cell lines, U87 and CHG-5. We found that the RACK1 expression was significantly higher in glioma tissues and cell lines when compared to normal brain tissues. It is worth noting that the expression of RACK1 was gradually upregulated with the malignancy of glioma, indicating that RACK1 is associated with the development and progression of gliomas, and hence may become a promising therapeutic target for this cancer.

Based on these clinical findings, we speculated that forced downregulation of RACK1 may suppress the development of gliomas. To test this hypothesis, RACK1-specific siRNA was applied to successfully downregulate the RACK1 expression in human glioma U87 and CHG-5 cells. As expected, RACK1 downregulation significantly inhibited the proliferation and invasion in U87 and CHG-5 cells. The Bcl-2 family plays an essential role in the regulation of cell survival and apoptosis, including both pro-apoptotic members such as Bax, as well as anti-apoptotic members such as Bcl-2. The balance between Bax and Bcl-2 determines the susceptibility of cells to the apoptotic signal (20). We found that siRNA-induced RACK1 downregulation notably promoted cell apoptosis of U87 and CHG-5 cells, partly through suppressing Bax expression and upregulating Bcl-2 expression, and hence breaking this balance for maintaining cell survival.

Based on these findings in vitro, we further applied tumor xenograft animal models to test whether RACK1 downregulation has an inhibitory effect on tumor growth in vivo, and found that stable downregulation of RACK1 in human glioma U87 cells markedly suppressed the tumor growth in vivo. These findings suggest that RACK1 may play a crucial role in tumorigenesis and progression of glioma in vitro and in vivo.

Src is a protein tyrosine kinase and participates in the regulation of multiple cellular processes including cell survival, proliferation and migration $(21,22)$. Accumulating evidence has revealed that Src could be recruited by RACK1, and acts as an oncogene in some types of cancer including glioma (23-26). Akt, also known as protein kinase B (PKB), acts in downstream of Src and plays an important role in the regulation of cell survival, proliferation and migration $(27,28)$.
However, whether Src/Akt signaling activity is involved in RACK1-mediated glioma development has yet to be investigated. The present study demonstrated that forced RACK1 downregulation suppressed the activity of Src/Akt signaling pathway in U87 and CHG-5 cells. These findings suggest that siRNA-induced RACK1 downregulation inhibits glioma development partly via suppressing Src/Akt signaling activity. Our results are consistent with the findings of Mamidipudi and Cartwright (29) that RACK1 promotes mitochondrial cell death and blocked Akt-mediated cell survival, partly via suppressing Src activity, in colon cancer cells, indicating that this regulatory mechanism may exist in multiple types of cancer cells.

In conclusion, the present study indicated that the upregulation of RACK1 is a common event in glioma, and that RACK1 plays a critical role for glioma development and progression in vitro and in vivo. Moreover, the underlying mechanism involves RACK1-mediated SRC/Akt signaling activity. Thus, the present study suggests that RACK1 may be a novel promising therapeutic target for glioma treatment.

\section{References}

1. Stewart LA: Chemotherapy in adult high-grade glioma: a systematic review and meta-analysis of individual patient data from 12 randomised trials. Lancet 359: 1011-1018, 2002.

2. Zhu VF, Yang J, Lebrun DG and Li M: Understanding the role of cytokines in Glioblastoma Multiforme pathogenesis. Cancer Lett 316: 139-150, 2012.

3. Sathornsumetee S, Reardon DA, Desjardins A, Quinn JA, Vredenburgh JJ and Rich JN: Molecularly targeted therapy for malignant glioma. Cancer 110: 13-24, 2007.

4. Linz U: Commentary on effects of radiotherapy with concomitant and adjuvant temozolomide versus radiotherapy alone on survival in glioblastoma in a randomised phase III study: 5-year analysis of the EORTC-NCIC trial (Lancet Oncol 2009;10:459-466). Cancer 116: 1844-1846, 2010.

5. Pulkkanen KJ and Yla-Herttuala S: Gene therapy for malignant glioma: current clinical status. Mol Ther 12: 585-598, 2005.

6. Dorn GW 2nd and Mochly-Rosen D: Intracellular transport mechanisms of signal transducers. Annu Rev Physiol 64: 407-429, 2002.

7. Adams DR, Ron D and Kiely PA: RACK1, a multifaceted scaffolding protein: structure and function. Cell Commun Signal 9: $22,2011$.

8. Wan L, Xie Y, Su L, Liu Y, Wang Y and Wang Z: RACK1 affects morphine reward via BDNF. Brain Res 1416: 26-34, 2011.

9. Battaini F, Pascale A, Paoletti R and Govoni S: The role of anchoring protein RACK1 in PKC activation in the ageing rat brain. Trends Neurosci 20: 410-415, 1997.

10. Marqués N, Sesé M, Cánovas V, et al: Regulation of protein translation and c-Jun expression by prostate tumor overexpressed 1. Oncogene: Mar 04, 2013 (Epub ahead of print). doi: 10.1038/onc.2013.51.

11. Guo Y, Wang W, Wang J, et al: Receptor for activated C kinase 1 promotes hepatocellular carcinoma growth by enhancing mitogenactivated protein kinase kinase 7 activity. Hepatology 57: 140-151, 2013.

12. Cao XX, Xu JD, Xu JW, et al: RACK1 promotes breast carcinoma migration/metastasis via activation of the RhoA/Rho kinase pathway. Breast Cancer Res Treat 126: 555-563, 2011.

13. Trerotola M, Li J, Alberti S and Languino LR: Trop-2 inhibits prostate cancer cell adhesion to fibronectin through the $\beta 1$ integrin-RACK1 axis. J Cell Physiol 227: 3670-3677, 2012.

14. Wu J, Meng J, Du Y, et al: RACK1 promotes the proliferation, migration and invasion capacity of mouse hepatocellular carcinoma cell line in vitro probably by PI3K/Rac1 signaling pathway. Biomed Pharmacother 67: 313-319, 2013.

15. Ruan Y, Sun L, Hao Y, et al: Ribosomal RACK1 promotes chemoresistance and growth in human hepatocellular carcinoma. J Clin Invest 122: 2554-2566, 2012.

16. Li G, Ji XD, Gao H, et al: EphB3 suppresses non-small-cell lung cancer metastasis via a PP2A/RACK1/Akt signalling complex. Nat Commun 3: 667, 2012. 
17. Lu F, Zhang $\mathrm{C}, \mathrm{Wu} \mathrm{WJ}$ and $\mathrm{Wu} \mathrm{YM}$ : RACK1 downregulation suppresses migration and proliferation of neuroblastoma cell lines. Oncol Rep 27: 1646-1652, 2012.

18. Li J, Guo Y, Feng X, et al: Receptor for activated C kinase 1 (RACK1): a regulator for migration and invasion in oral squamous cell carcinoma cells. J Cancer Res Clin Oncol 138 563-571, 2012.

19. Wang F, Osawa T, Tsuchida R, Yuasa Y and Shibuya M: Downregulation of receptor for activated C-kinase 1 (RACK1) suppresses tumor growth by inhibiting tumor cell proliferation and tumor-associated angiogenesis. Cancer Sci 102: 2007-2013, 2011.

20. van Delft MF, Wei AH, Mason KD, et al: The BH3 mimetic ABT-737 targets selective Bcl-2 proteins and efficiently induces apoptosis via Bak/Bax if Mcl-1 is neutralized. Cancer Cell 10 389-399, 2006.

21. Gelman IH: Src-family tyrosine kinases as therapeutic targets in advanced cancer. Front Biosci 3: 801-807, 2011.

22. Aleshin A and Finn RS: SRC: a century of science brought to the clinic. Neoplasia 12: 599-607, 2010
23. Doan AT and Huttenlocher A: RACK1 regulates Src activity and modulates paxillin dynamics during cell migration. Exp Cell Res 313: 2667-2679, 2007.

24. Huveldt D, Lewis-Tuffin LJ, Carlson BL, et al: Targeting Src family kinases inhibits bevacizumab-induced glioma cell invasion. PLoS One 8: e56505, 2013.

25. Stedt H, Alasaarela L, Samaranayake H, et al: Specific inhibition of SRC kinase impairs malignant glioma growth in vitro and in vivo. Mol Ther Nucleic Acids 1: e19, 2012.

26. Creedon $\mathrm{H}$ and Brunton VG: Src kinase inhibitors: promising cancer therapeutics? Crit Rev Oncog 17: 145-159, 2012.

27. Sheppard K, Kinross KM, Solomon B, Pearson RB and Phillips WA: Targeting PI3 kinase/AKT/mTOR signaling in cancer. Crit Rev Oncog 17: 69-95, 2012.

28. Hemmings BA and Restuccia DF: PI3K-PKB/Akt pathway. Cold Spring Harb Perspect Biol 4: a011189, 2012

29. Mamidipudi V and Cartwright CA: A novel pro-apoptotic function of RACK1: suppression of Src activity in the intrinsic and Akt pathways. Oncogene 28: 4421-4433, 2009. 